\title{
El número áureo como elemento motivador hacia el estudio de las matemáticas
}

\section{The golden number as a motivating element towards the study of mathematics}

\author{
Dorenis Josefina Mota Villegas \\ https://orcid.org/0000-0002-2290-986X \\ Ricardo Enrique Valles Pereira \\ https://orcid.org/0000-0002-0873-9833 \\ Pontificia Universidad Católica del Ecuador, Ecuador
}

Autor por correspondencia: dorenismota@gmail.com; prfricardovalles@gmail.com

Fecha de recepción: 14 de octubre de 2019 - Fecha de aceptación: 20 de abril de 2020

\section{Resumen}

El presente artículo muestra la descripción y resultados preliminares de una experiencia llevada a cabo en una clase matemática básica; la cual tuvo como propósito principal motivar a los estudiantes al estudio de esa Ciencia. La experiencia se basó en la implementación de un proyecto pedagógico fundamentado en el origen, evolución, desarrollo y aplicación del número áureo; este tema fue seleccionado cuidadosamente por su conexión directa con elementos de la realidad, siendo éste el factor detonante para despertar el interés del estudiante por las matemáticas. Metodológicamente la modalidad de la investigación fue de acción participativa; con la previa revisión bibliográfica de teorías relacionadas tanto con el número áureo como con el proceso de enseñanza y aprendizaje de la matemática incluyendo importancia de la motivación en el desarrollo óptimo de dicho proceso. Los sujetos de estudio fueron los estudiantes del primer nivel cursantes de Matemática Básica para el período académico 2018 II. Los resultados preliminares obtenidos arrojaron una aceptación favorable de la asignatura ya que la mayoría de los estudiantes se mostraron receptivos y motivados a la realización de las actividades y manifestaron sentirse satisfechos con el aprendizaje obtenido.

Palabras Claves: número áureo; motivación; enseñanza; matemática

\begin{abstract}
This paper shows the description and preliminary results of an experience carried out in a basic mathematical class; which had as main purpose to motivate students to study that Science. The experience was based on the implementation of a pedagogical project based on the origin, evolution, development and application of the golden number; this theme was carefully selected for its direct connection with elements of reality, this being the trigger to arouse the student's interest in mathematics. Methodologically, the research modality was participatory action; with the previous literature review of theories related to both the golden number and the teaching and learning process of mathematics, including the importance of motivation in the optimal development of said process. The subjects of study were the first level students attending Basic Mathematics for the academic period 2018 II. The preliminary results obtained showed a favorable acceptance of the subject since most of the students were receptive and motivated to carry out the activities and said they were satisfied with the learning obtained.
\end{abstract}


Key words: golden number; motivation; teaching; mathematics

\section{Introducción}

Es un hecho que en la actualidad la Matemática forma parte del programa obligatorio de educación en todos los países latinoamericanos, y ese carácter formal de va desde los primeros niveles de escolaridad (primaria) hasta los últimos niveles de desarrollo profesional acreditado (Universidad). En este último escenario, el universitario, se puede mencionar que, aunque muchas carreras no estén vinculadas de forma directa con la Matemática pura, existen unos conocimientos elementales que deben ser adquiridos por los estudiantes y entonces, se incluyen en las mallas curriculares asignaturas de Matemática básica afines a todas las carreras y modalidades de estudio.

En la Pontificia Universidad Católica del Ecuador, una Universidad particular con sede principal en Quito, Ecuador; donde, si bien es cierto que se estudian carreras afines a la Matemática de forma directa (Ingeniería, por ejemplo), también existen otras carreras donde la relación de la Matemática no está determinada de forma explícita (Arquitectura, Diseño y Artes); hay asignaturas comunes, que los estudiantes, independientemente de las carreras que cursen, tendrán que estudiar de forma obligatoria, tal es el caso de Matemática, impartida en el primer nivel a los estudiantes de recién ingreso para la carrera de Diseño Gráfico y Diseño de Productos, los cuales están insertados en la Facultad de Arquitectura Diseño y Artes. En relación con esta asignatura: Matemática, muchos de los estudiantes manifiestan sentir poca motivación e, incluso, aversión o rechazo hacia el estudio de la misma, incluso, aquellos estudiantes que entran a la Universidad para cursar carreras afines a la ingeniería también manifiestan, en muchos casos, tener una mala experiencia con el estudio de la asignatura en el nivel académico anterior, es decir la secundaria o el bachillerato (Padrón, 2016) (Caballero - Jiménez \& Espínola - Reyna, 2016) y es que, de forma general, la literatura actual muestra, la creciente preocupación de investigadores en el área durante los últimos años por el bajo rendimiento, la deserción y la disminución de la cantidad de estudiantes que quieren seguir carreras afines a esta Ciencia y los intentos que se han hecho por remediar la situación desde diferentes perspectivas de carácter pedagógico (Cerda, 2018) (Fajardo Bullón, Maestre Campos, Felipe Castaño, León del Barco, \& Polo del Río, 2017) (Delgado, 2017); (Ramohapi, 2017) (Banerjee, 2016) (PISA, 2016).

Los estudiantes continuamente manifiestan que el estudio de las matemáticas se hace tedioso y de poco interés debido a que generalmente no logran vincular cuál es la relación que tiene la Ciencia con su entorno o con su realidad, preguntándose de forma reiterada cuál es la ocupación que le dará a esos conocimientos, y por qué es tan necesario y obligatorio aprenderlos. Al respecto, (Mora, 2009) comenta que esta concepción de las matemáticas escolares, se vuelve un aprendizaje incomprendido debido a su forma estructuralista y formal de impartirse. En consecuencia, se enseñan matemáticas descontextualizadas, acabadas, cuya conexión con la realidad, o sea, con su cotidianidad fuera del aula es nula; estas razones, entre otras, serian algunas causas de la apatía, desmotivación, poco interés y el cuestionamiento constante hacia el aprendizaje obligatorio de tópicos elementales de las matemáticas que el estudiante debe poseer a nivel universitario.

Por lo anteriormente mencionado, se ha planteado e implementado un proyecto, dirigido a estudiantes del Primer Semestre de la Pontificia Universidad Católica del Ecuador, 
matriculados en el Período 2018-II, la cual busca vincular las matemáticas con la realidad, y ese vínculo se ha realizado mediante la conexión que ha tenido (perspectiva histórica) y tiene (aplicaciones en la actualidad): el número áureo que puede observarse y aplicarse en diversas áreas del saber tales como naturaleza, arte, música, arquitectura, diseño. En ese sentido, apoyados en ese vínculo se procuró llamar la atención del estudiante por el estudio de las Matemáticas.

En ese orden de ideas, el objetivo principal fue motivar al discente al estudio de las matemáticas usando como elemento atractivo al número áureo por sus propiedades matemáticas y su relación con otras Ciencias.

\section{Metodología}

El presente trabajo se corresponde con una experiencia de aula enmarcada en una investigación acción (Burns, 2011) donde se evaluaron resultados preliminares del proceso aplicado; cabe aclarar, no se considera la experiencia como un proceso cerrado o unidireccional, ya que constantemente se aplica, ajusta y reaplica; y se evalúan y reevalúan los resultados que se consideran cambiantes en todo momento. En cuanto al diseño, fue de campo, ya que, según (Perez, 2009), la información se recopila directamente de la realidad, fiel a cómo sucedieron los acontecimientos, apoyados en material bibliográfico pertinente y actualizado que dieron soporte teórico a los hallazgos encontrados.

En cuanto al contexto y sujetos de la investigación, la experiencia de aula tuvo lugar en la Pontificia Universidad Católica de Ecuador, Sede Quito, Ecuador, donde se desarrollaron actividades relacionadas con el número áureo (generalidades, historia, aplicaciones intra y extra matemáticas) como elemento motivador hacia el estudio de la matemática.

Dichas actividades fueron aplicadas a 31 estudiantes de la Facultad de Arquitectura, Diseño y Artes (FADA) inscritos en la asignatura de Matemática (2do Nivel) afines a las carreras de Arquitectura y Diseño, matriculados en el periodo académico 2018 II.

\section{Resultados y discusión}

Antes de dar inicio a los resultados de investigación, se considera de relevancia presentar las conclusiones teóricas obtenidas de la revisión bibliográfica exhaustiva sobre los diferentes elementos que dieron lugar a este estudio, entre esos elementos están la relación que existe entre la motivación y el aprendizaje de las matemáticas que permitió dimensionar el problema presente que dio origen al estudio; la Teoría Socio epistemológica de la Matemática Educativa (TSME) que permitió identificar desde el punto de vista de la Didáctica de las matemáticas la postura a seguir para darle respuesta al problema y el número áureo y didáctica como eslabón o el elemento conector entre los estudiantes y las matemáticas.

\section{Motivación y aprendizaje de las Matemáticas}

El objetivo fundamental de presentar los tópicos matemáticos, en las aulas de clase, es apoyar al estudiante para que adquiera y desarrolle la comprensión y aplicación de los conceptos y procedimientos matemáticos; adicionalmente los dicentes deberían evidenciar 
que las matemáticas son de utilidad en su entorno, tanto en lo educativo como en el ámbito profesional y por qué no, en su entorno cotidiano (Zemelman, 1998).

Para que el estudiante se apropie de los contenidos matemáticos adecuadamente, no solo hace falta que tenga aptitudes, sino que también debe poseer actitudes favorables hacia el estudio de las mismas; desde hace más de una década (Nuria Gil, 2005) ya se mencionaba la importancia del componente afectivo dentro del aprendizaje de las matemáticas, la cual ha sido respaldada por innumerables trabajos desarrollados en los últimos años con rigor científico sobre el tema, donde en muchos de ellos se demuestra la influencia de la actitud hacia la matemática tomando como referencia el rendimiento escolar, el auto concepto matemático y la ansiedad hacia el estudio de las ciencias. La vinculación está dada en que a medida que se tiene una buena actitud se tiene un mejor auto concepto y baja la ansiedad; recíprocamente, si se tiene una elevada ansiedad y mala actitud el auto concepto matemático no será bueno y estos elementos pueden influir directamente sobre el rendimiento del estudiante en matemática.

En el mismo orden de ideas, (Angulo, 2006) considera que al enseñar matemáticas, se debe proporcionar paralelamente los medios de reflexión para valorar y disciplinar estructuras cognoscitivas relacionadas con un marco referencial de orden platónico, dentro de un ambiente rígido, formal, donde se relacionan objetos no ostensibles(los objetos matemáticos solo pueden ser representados a través del lenguaje simbólico y gráfico, ya que no poseen propiedades físicas, como otros objetos como por ejemplo una mesa, una silla, por lo tanto su construcción es netamente representacional). Sostiene este mismo autor que las matemáticas,

[...] generalmente, suele simbolizarse como un contexto axiomático formalizado. En ellos se articula el rigor del discurso formal que nace de las puras relaciones de los objetos; de allí, se edifica la estructura matemática que se levanta a juicio de los razonamientos lógicos deductivos. Quien aprende, desea voluntariamente ensayar de forma creativa encadenamientos racionales en el juego del discurso formal para transformarlo en implicaciones lógicas libres de contradicciones y conforme a la estructura axiomática de referencia. De este modo, se pueden encarar eficientemente problemas que demandan soluciones; bien sea, en el mundo de las ideas o en el mundo de los hechos. Esta perspectiva es netamente formalista. Su solidez se centra en el número finito de razonamientos deductivos y sujeto a las pruebas de consistencia absolutas propuesta por Gödel, en que sostiene la no contradicción e incompletitud de sus proposiciones inscritas en los sistemas formales que se apuntalan por los recursos de la aritmética. (pág. 343).

Sin embargo, está la contraparte de la matemática formal, que es aquella que tiene que ser enseñada con sentido práctico, y no se trata de disminuir la formalidad o la rigurosidad propia de la Ciencia, sino de enseñarse desde la aplicación bajo el argumento de mostrarse interesante y significativa para el discente, es decir, los resultados deben ser útiles a la luz del contexto del estudiante; por lo tanto, la enseñanza debe estar orientada hacia una matemática aplicada, que, además, debe cambiar conforme se modifique el contexto del estudiante, e incluso, deberá adaptarse a los cambios producidos a lo largo del tiempo; donde los seres humanos buscan la superación en su sobrevivencia y las matemáticas ofrecen la oportunidad de cambiar o crear una mejora en su entorno (Carraher, 1982) (Salinas, 2009).

Esta obra se comparte bajo la licencia Creative Common Atribución-No Comercial 4.0 International (CC BY-NC 4.0)

Revista de la Universidad Internacional del Ecuador. URL: https://www.uide.edu.ec/ 
Lo mencionado anteriormente busca contrarrestar la concepción que los estudiantes, en su mayoría, tienen de la Ciencia, para ellos las matemáticas es un conocimiento acabado, no discutible, no cuestionable, que solo tiene existencia dentro del aula de clases y no es capaz de trascender fuera del contexto escolar ni tampoco de relacionarse con otras ciencias, precisamente, por esa forma estructuralista y rígida que tradicionalmente tenemos de enseñarla.

Sobre la base de esos preceptos, este proyecto buscó romper ese paradigma, esa forma de pensar negativa hacia las matemáticas; es hacer que la mayor cantidad de estudiantes puedan interesarse de forma positiva, con una motivación adecuada, al estudio de dicha Ciencia. Adicionalmente un elemento importante a nuestro favor es el cambio de nivel o la ruptura que supone pasar de la Secundaria a la Universidad, donde los prejuicios pueden quedar atrás por tratarse del inicio de una nueva etapa estudiantil y se pueda asumir una actitud diferente y positiva, centrada en la relación o vínculo que existe entre las matemáticas y todo lo que nos rodea, y que consideramos, un elemento ideal de motivación: la relación que el número áureo tiene con la realidad, tal como veremos de forma resumida en un posterior apartado.

\section{Teoría Socio epistemológica de la Matemática Educativa (TSME)}

Actualmente, existen diversas teorías inmersas en la Didáctica de la Matemática como Ciencia, las cuales surgen de estudios realizados por numerosos investigadores y expertos en el área de la Didáctica de la Matemática quienes han direccionado sus esfuerzos a responder preguntas propias de la Matemática Educativa como, por ejemplo: ¿Si la matemática es una actividad netamente humana, por qué cada vez menos humanos quieren estudiarla y cultivarla? ¿Cómo puede promoverse el estudio de la matemática de tal manera que podamos ir sumando cada vez más seguidores y no detractores? ¿Cuáles son los mecanismos didácticos necesarios para que las personas, no afines, a la matemática, puedan aprehender al menos el conocimiento mínimo matemático aceptado desde el punto de vista profesional? En diferentes direcciones, pero con un mismo propósito: hacer que la matemática sea cada vez más accesible a la mayoría de las personas; se han desarrollado varias Teorías relacionadas con todo lo que implica el proceso de enseñanza y aprendizaje de esta Ciencia en pro de responder las cuestiones descritas en el párrafo anterior; una de ellas es Socio epistemología de la Matemática Educativa (TSME) la cual, si bien es cierto, no es la única teoría sobre la Didáctica de la Matemática, es la que sustentó, desde el punto de vista didáctico nuestro Proyecto, ya que para en su realización y ejecución consideramos los aspectos descritos en dicha teoría y de los cuales haremos un breve resumen en los párrafos siguientes.

La TSME fue promovida por Cantoral: (Cantoral, Categorías relativas a la apropiación de una base de significaciones para conceptos y procesos matemáticos de la Teoría elemental de las Funciones Analíticas. Simbiosis y Predación entre las nociones de "el Prædiciere" y "lo Analítico". (Tesis Doctoral), 1990) (Cantoral, Matemática, Matemática Escolar y Matemática Educativa., 1995) (Cantoral, La sensibilidad a la contradicción: Un estudio sobre la noción de logaritmo de, 2002) (Cantoral, Fundamentos y métodos de la Socioepistemología, 2011) (Cantoral, Teoría Socioepistemológica de la Matemática Educativa. Estudios sobre construcción social del conocimiento, 2013). quien es el principal investigador y desarrollador de la misma hasta la actualidad, y a la cual se han sumado numerosos colaboradores (Cantoral \& Farfán, Matemática Educativa: Una visión de su 
evolución, 2003), (Cantoral, Reyes-Gasperini, \& Montiel, Socioepistemología, Matemáticas y Realidad, 2014) y tiene como finalidad "democratizar el aprendizaje de las matemáticas" (p. 96). Para ello trata de dar respuesta a preguntas principales: “¿cómo lograr que disfruten y entiendan las matemáticas la mayoría de estudiantes de una clase?" (p. 112), para lo cual plantea estrategias dirigidas a lograr ese objetivo basadas en intervenciones didácticas no tradicionales fundamentadas en los siguientes tres aspectos: Aula extendida, el saber en tanto conocimiento en uso y una visión crítica, solidaria y humanística de la sociedad del conocimiento.

Esta teoría tiene sus orígenes en el Sur del continente americano, con la intencionalidad de dar un carácter propio a las investigaciones desde y para Latinoamérica, como una manera de rescatar la identidad regional y de crear soluciones específicas a situaciones particulares en el marco del contexto socioeconómico y cultural de cada País latinoamericano (Cordero Osorio \& Silva-Crocci, 2012). No obstante, en la actualidad, esta teoría de la Didáctica de la Matemática se ha extendido en el ámbito internacional de forma paulatina, lo que favorece a que pase de ser una teoría emergente a una teoría en etapa de consolidación.

Los aspectos de la TSME que fueron considerados para la experiencia aquí relatada son los descritos a continuación en palabras de (Cantoral, Reyes-Gasperini, \& Montiel, Socioepistemología, Matemáticas y Realidad, 2014):

-Las matemáticas, como parte de la cultura, se construyen a partir de vivencias cotidianas de los individuos a través de prácticas socialmente compartidas. -El desarrollo de una manera matemática de pensar entre la población exige de una "descentración del objeto" y de la incorporación de las prácticas que le acompañan. Es importante precisar en este sentido, que se debe asumir la legitimidad de toda forma de saber, sea este popular, técnico o culto, pues en su conjunto constituyen la sabiduría humana.

-El discurso Matemático Escolar vigente, debe rediseñarse, pues se trata de un sistema de razón de carácter hegemónico que legitima la imposición de significados, tanto a estudiantes como a docentes cuando reduce el saber al conocimiento segmentado, despersonalizado y descontextualizado. Dicho discurso, dominante en las aulas de hoy día, excluye al docente de la acción creativa y lo reduce a la figura de "facilitador" de técnicas y procedimientos. -El rediseño del discurso Matemático Escolar, precisa de un proceso de empoderamiento docente que permita a las y los profesionales de la enseñanza apropiarse del saber que enseñan a fin de profesionalizar la gestión educativa mediante la producción de diseños situados de intervención pedagógica que problematizan al saber matemático escolar.

-La dualidad individuo - colectividad se supera con la anidación de prácticas que parten de la acción del individuo sobre el medio, se constituyen en actividades humanas que, reiteradas con intencionalidad, devienen en prácticas socialmente compartidas en el contexto de grandes prácticas de referencia. Todas ellas, normadas por prácticas sociales que caracterizan al colectivo y al campo de saber específico. (pág. 112-113).

Se puede sintetizar entonces la necesidad de reestructurar las prácticas educativas tradicionales para la enseñanza de las matemáticas, básicamente, si queremos obtener buenos 
resultados en el aprendizaje, debemos cambiar la forma de enseñarla. Adicionalmente, la TSME plantea la creación de modelos propios para ser aplicables de acuerdo al contexto donde se desarrolla la actividad matemática.

La TSME parte de que las matemáticas, debe surgir de la práctica y no del concepto, como generalmente se hace, esa práctica a su vez viene del contexto, por lo tanto, la adquisición de las nociones matemáticas conceptuales son últimas en ese proceso. Esto se logra a partir de un docente que conozca el contexto de sus estudiantes y saque provecho de los elementos matemáticos que pueden surgir de esa cotidianidad. En el caso particular que nos atañe, se cuenta con una población estudiantil joven (17-22 años) que ingresa a la Universidad bajo la modalidad Presencial de Enseñanza-Aprendizaje, la cual se ha desarrollado en un ambiente urbano (Quito, Capital de Ecuador) acostumbrados al mundo globalizado bajo la influencia de las Nuevas Tecnologías (acceso a teléfono inteligente, uso frecuente de redes sociales, entre otros) y bajo esa perspectiva, procuramos generar escenarios familiares para ellos que, a su vez, favorezcan una nueva forma de hacer matemáticas, la cual vincula al número áureo con actividades y situaciones cotidianas.

\section{Número áureo y Didáctica}

La relación existente entre el número irracional phi, también llamado número áureo o número de oro $(\varphi)$ con áreas intra-matemáticos (sucesión de Fibonacci, el rectángulo áureo, la estrella pitagórica) y extra-matemática (arquitectura, diseño, biología, botánica, ecología, entre otras) ha sido ha sido estudiada y difundida desde tiempos inmemoriales. Son innumerables las aplicaciones del número áureo desde la antigüedad, por ejemplo, Pitágoras ya conocía la relación de este número con algunas formas geométricas como la estrella pitagórica; estuvo la proporción áurica presente en la construcción de obras antiguas importantes como el Partenón y fue empleada por artistas de renombre como Da Vinci y Dalí para crear sus obras más conocidas a nivel mundial como la Gioconda y la Leda Atómica respectivamente (Ghyka, 1977) (Doczi, 1994); (Livio, 2002) (Hemenway, 2008) (Corbalán, 2012).

A partir de la publicación de la obra de Luca Pacioli en 1949 y titulada: El tratado de la divina proporción; muchas son las aplicaciones que se ha dado a la proporción áurea desde ese entonces hasta la actualidad y es que; sin duda alguna, esa conexión, que muchos afirman, tiene la proporción áurea con la belleza, ha despertado el interés no solo de matemáticos sino de otros eruditos de ramas diferentes del saber; interés que se mantiene hasta nuestros días y prueba de ello es la extensa información que puede extraerse desde los diferentes medios actuales de información (libros, revistas, páginas de internet) y los eventos que se desarrollan año tras año, con el único propósito de divulgar las últimas aplicaciones del número áureo como lo es la International Conference on Fibonacci Numbers y la publicación periódica de la revista The Fibonacci Quaterly.

En el caso particular de la experiencia de aula trabajada, si bien es cierto que el estudio del número de oro no es parte explícita de los tópicos que deben ser abordados en la asignatura Matemática Básica, si está íntimamente relacionado con los temas que allí se abordan (números irracionales, raíces de polinomio de grado 2, sucesiones, geometría básica, proporciones, entre otros) y es, desde esa perspectiva, que se involucró al estudiantado a tener un contacto cercano con la Matemática, partiendo del hecho de la relación evidente de la 
proporción áurea con su entorno (relación que el estudiante debió comprobar mediante las actividades realizadas) y luego del vínculo entre dicho número y muchos de los tópicos abordados en clase; en tal sentido, el proyecto no interrumpió el contenido que debió ser abordado en la asignatura de Matemática Básica, por el contrario fue un medio de apoyo y motivación para la introducción de muchos de los temas estudiados.

En el siguiente apartado se mencionan, en detalle, los contenidos abordados sobre el número áureo y cómo estuvieron distribuidas las actividades para el desarrollo de cada contenido.

\section{Descripción de la experiencia}

Bajo el contexto antes mencionado, la primera actividad que se le asignó al estudiantado fue la de obtener información sobre el número de oro y sus generalidades: la razón áurea, el rectángulo áureo, la sucesión de Fibonacci y su relación con el número de oro. Como inducción al tema, también se le sugirió la visualización de un vídeo de libre acceso ubicado en la plataforma YouTube (Ventura, 2016), de duración corta (3:55 minutos), ideal para los nativos digitales (Prensky, 2011), es decir, para el perfil de los sujetos de estudio por su corta duración y precisión del contenido; con esta información disponible, los estudiantes desarrollaron en el aula de clases las siguientes actividades, de acuerdo al cronograma planificado:

\section{Actividad 1: Mesa de discusión}

Con el material previamente investigado, se hizo una distribución grupal de los estudiantes en el aula de clases, donde se les indica que deben realizar una reconstrucción histórica y epistemológica del número áureo en base al análisis e interpretación del material recopilado por cada integrante del equipo.

\section{Actividad 2: Demostración del número áureo, construcción del rectángulo áureo y de la espiral de Durero}

Esta actividad es realizada principalmente por el docente con la colaboración de los estudiantes; el docente demuestra la proporción áurea y obtiene de ella el número de oro $(\varphi)$ a partir de la construcción de un rectángulo áureo; luego construye rectángulos áureos sucesivos hasta formar la espiral de Durero, los estudiantes lo acompañan en todo el proceso de forma participativa.

\section{Actividad 3: Mapa mental}

En un siguiente encuentro, los estudiantes realizaron mapas mentales con los registros bibliográficos de la reconstrucción histórica y epistemológica del número áureo, incluyendo la relación de dichos aportes con la naturaleza. En tal sentido, las estructuras de los mapas se centraron en encontrar y ubicar la íntima relación que tiene el número de oro con diferentes elementos de la naturaleza. Cabe destacar que para la evaluación de dichos mapas se siguió una rúbrica evaluativa que previamente se le había presentado al estudiante, junto con las asignaciones de la actividad. 
A manera de ilustración en la Ilustración \# 1 se presentan algunas imágenes de los mapas mentales realizados por los estudiantes:

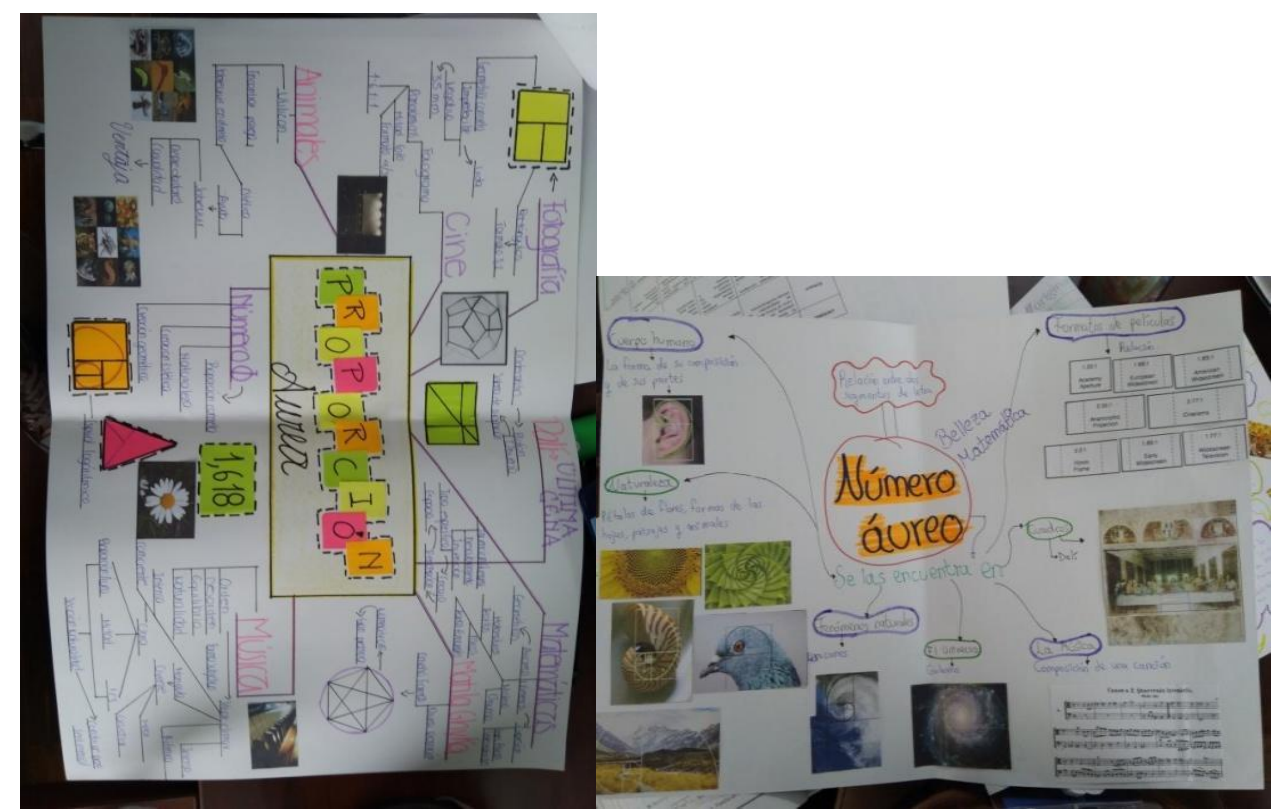

Ilustración \# 1 Mapas mentales realizados por los estudiantes de arquitectura sobre el número áureo y sus aplicaciones

\section{Actividad 4: El número de oro en diseño, construcción y arquitectura: Época antigua, Época moderna}

Los estudiantes realizan una investigación específica sobre el diseño, la construcción y la arquitectura relacionada con el número de oro (época antigua, época moderna) en el escenario regional (Latinoamérica) y local (Ecuador).

\section{Actividad 5: Realización de maqueta}

Una vez realizadas las actividades anteriores, el estudiante realizó una maqueta de alguna obra arquitectónica ya conocida o creada por él, empleando para dicha construcción la proporción áurea. A continuación ver la Ilustración \# 2 donde se presentan algunas evidencias fotográficas de las maquetas realizadas.

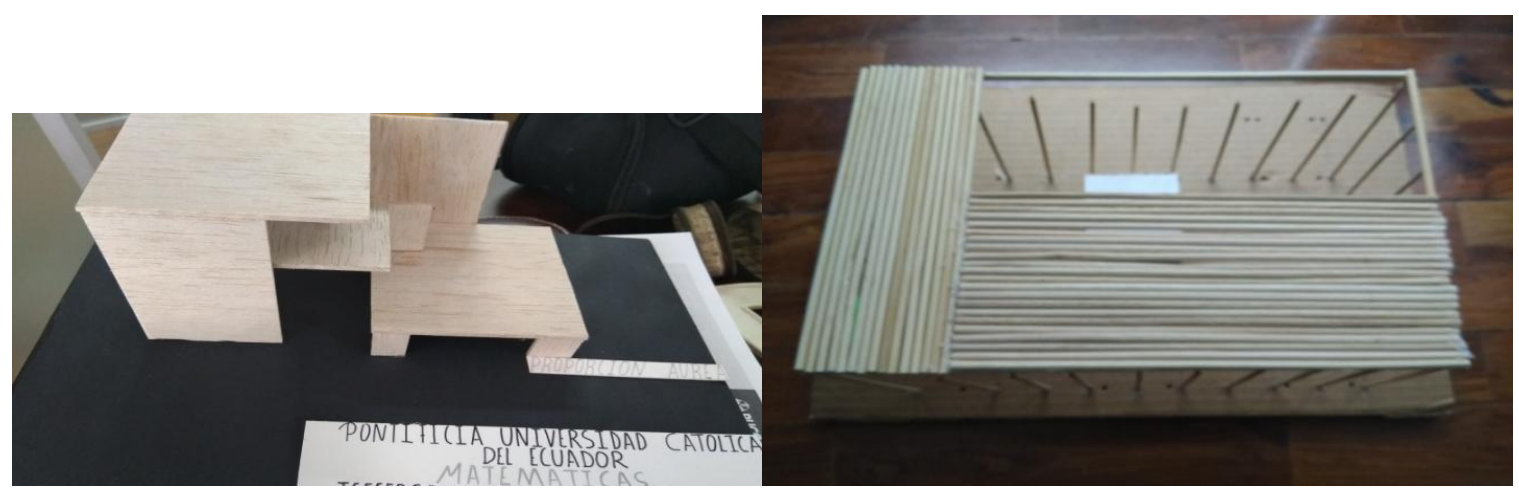

Ilustración \# 2 Maquetas realizadas por los estudiantes de arquitectura sobre construcciones inspiradas en el número áureo 


\section{Resultados preliminares}

A continuación se da a conocer una muestra general de los resultados obtenidos en base a la información recopilada (encuesta) sobre las actividades aplicadas a los alumnos del curso de Matemática para Arquitectura del segundo nivel de la Pontificia Universidad Católica del Ecuador.

\section{Muestra del análisis estadístico de algunas de las preguntas realizadas}

Pregunta 1: ¿Consideras que mediante el estudio del número áureo pudiste comprender algunos conceptos de matemática básica?

Tabla. 1

Resultados de las respuestas a la pregunta 1

\begin{tabular}{lll}
\hline Respuesta & Cantidad de estudiantes & Porcentaje (\%) \\
\hline Sí & 22 & 71 \\
No & 2 & 6 \\
Nula & 7 & 23 \\
Total & 31 & 100 \\
\hline \multicolumn{2}{c}{ Fuente: elaboración propia }
\end{tabular}

En la Tabla 1 (ver Tabla 1) se describe que más de la mitad de los encuestados representada por el $71 \%$ respondieron que el estudio del número áureo les permitió adquirir conceptos de matemática básica, y entre esos conceptos los estudiantes señalaron: operaciones elementales, los números irracionales, proporciones, medición, sucesiones, polígonos y poliedros regulares y transformaciones geométricas. Por su parte el $6 \%$ de los estudiantes señaló que no logró comprender conceptos matemáticos básicos mediante el estudio del número áureo argumentando que el número áureo era complejo en sí mismo y los conceptos estudiados no eran básicos sino avanzados y poco comprensibles, finalmente un $23 \%$ de los encuestados fue considerado con respuestas nulas ya que estas carecieron de argumentación o justificación.

Pregunta 2: ¿Consideras que fueron de utilidad, como motivación positiva, las actividades realizadas sobre el número áureo para el estudio de la matemática básica? Justifica tu respuesta.

Tabla. 2

Resultados de las respuestas a la pregunta 2

\begin{tabular}{lll}
\hline Respuesta & Cantidad de estudiantes & Porcentaje $(\%)$ \\
\hline Sí & 28 & 93 \\
No & 3 & 7 \\
\hline Total & 31 & 100 \\
\hline \multicolumn{3}{c}{ Fuente: elaboración propia }
\end{tabular}

En la Tabla 1 (ver Tabla 1) se evidencia que gran parte de los encuestados, representada por el $93 \%$ de los estudiantes, afirma que son útiles las actividades realizadas sobre el número áureo como elemento motivador hacia el estudio de la matemática básica. En la parte abierta de la pregunta 2 donde se le pide al estudiante que señale el porqué de la respuesta, allí debió escribir la justificación de su respuesta y se evidenció la valoración positiva de las actividades realizadas a través de comentarios como: -no había pensado en la relación estrecha que tiene la matemática y el número áureo con mi Carrera, sin Esta obra se comparte bajo la licencia Creative Common Atribución-No Comercial 4.0 International (CC BY-NC 4.0) 
duda alguna lo aprendido me será de gran utilidad-, -la forma diferente de abordar la matemática desde sus aplicaciones y conexión con la realidad hicieron que me interesara en ella-, -para ser la una asignatura que no me gusta logré comprenderla y hasta disfrutarla, siga así-, -fue motivador ver por fin la matemática aplicada a la Carrera-, -antes de la asignatura no sabía nada del número áureo ni de su conexión con la arquitectura, ahora entiendo que la proporción áurea también se relaciona además con muchas otras cosas-; por su parte las valoraciones negativas representadas por el $7 \%$ restante de los encuestados estuvieron justificadas por: -la matemática me parece difícil y lo sigue siendo a pesar del número áureo, el número áureo complica más las cosas porque hay que investigar y pensar, prefiero solo ejercicios paso a paso y ya-, -profesora lo intentó, pero falté a clases y no pude entender bien lo que quiso hacer con el número áureo, me jale, retiraré la materia-. No hubo respuestas nulas o carentes de justificación para esta pregunta.

Pregunta 3: ¿Consideras que la matemática básica estudiada puede ser de beneficio o se relaciona con tu Carrera?

Tabla. 3

Resultados de las respuestas a la pregunta 3

\begin{tabular}{lll}
\hline Respuesta & \multicolumn{1}{c}{ Cantidad de estudiantes } & Porcentaje (\%) \\
\hline Sí & 25 & 80 \\
No & 3 & 10 \\
Nula & 3 & 10 \\
Total & 31 & 100
\end{tabular}

Elaboración propia a partir de los datos aportados por la encuesta aplicada

En la Tabla 3 (ver Tabla 3) se observa que la gran mayoría de encuestados representada por el $80 \%$ manifestaron estar de acuerdo en que la matemática estudiada es de beneficio o se relaciona con su Carrera, que en este caso es la carrera de Arquitectura, las justificaciones a esta respuesta se fundamentan en comentarios como: - se observó que así como está relacionado el número áureo con la Arquitectura lo está toda la matemática en sí, por las mediciones y todo lo relacionado que debemos hacer-, -debemos aprender los elementos de matemática básica para sacarle provecho en la arquitectura como lo hicieron los arquitectos del pasado y lo hacen los del presente-, -en cada medición, así no utilicemos necesariamente al número áureo, está la matemática presente en la arquitectura-, -la matemática está en todos lados, también en la arquitectura y nos ayudará a hacer mejor nuestro trabajo si la sabemos usar-. Sin embargo una minoría representada por el $10 \%$ manifestó que la matemática básica no es de relevancia para la carrera, dicha respuesta se basa esencialmente en argumentaciones como: -ya sabía la matemática básica necesaria para la Carrera antes de ver la asignatura-, -estudio arquitectura y no ingeniería civil para no tener que ver matemáticas-, -creo que lo esencial para la arquitectura es el buen trazo y no ser un genio en matemáticas-. El 10\% restante simplemente no justificó la respuesta emitida.

Pregunta 4: ¿Consideras que la matemática básica estudiada (apoyada en el número áureo), permitirá valorar a la Ciencia en el ámbito profesional a futuro? 
Tabla. 4

Resultados de las respuestas a la pregunta 4

\begin{tabular}{lll}
\hline & Cantidad de estudiantes & Porcentaje $(\%)$ \\
\hline Sí & 21 & 68 \\
No & 1 & 3 \\
Nula & 9 & 29 \\
Total & 31 & 100 \\
\hline \multicolumn{3}{c}{ Fuente: elaboración propia }
\end{tabular}

En la Tabla 4 (ver Tabla 4) se describe que veintiuno de los encuestados representado por el $68 \%$ respondieron que el desarrollo de los tópicos abordados en el curso de matemática básica apoyada en los tópicos del número áureo, les permitirá valorar a la Ciencia en su futuro ámbito profesional. Por su parte, un estudiante, representando el 3\% indicó que los tópicos del número áureo abordados en el curso de matemática básica, no le alcanza para valorar a la Ciencia en su futuro ámbito profesional. Finalmente, el29\% de los encuestados fue considerado con respuestas nulas ya que estas carecieron de argumentación o justificación.

Pregunta 5: ¿Consideras que la matemática básica estudiada (apoyada en el número áureo) puede fungir de sustento en las asignaturas prácticas de tu carrera?

Tabla. 5

Resultados de las respuestas a la pregunta 5

\begin{tabular}{lll}
\hline Respuesta & Cantidad de estudiantes & Porcentaje (\%) \\
\hline Sí & 26 & 84 \\
No & 3 & 10 \\
Nula & 2 & 6 \\
Total & 31 & 100 \\
\hline \multicolumn{3}{c}{ Fuente: elaboración propia }
\end{tabular}

En la Tabla 5 (ver Tabla 5) se describe que un 84\% de los encuestados respondieron que el estudio de los tópicos sobre el número áureo les servirá de apoyo en las asignaturas prácticas de su carrera. No obstante, un $10 \%$ de los estudiantes señaló que el estudio de los tópicos sobre el número áureo no les servirá de sustento en sus cátedras prácticas de la carrera; y finalmente un 6\% de los encuestados fue considerado con respuestas nulas ya que estas carecieron de argumentación o justificación.

Pregunta 6: ¿Consideras que el estudio de las asignaturas de matemática superior, deberían ser abordadas con la metodología implementada en el curso de matemática básica, fundamentada en los tópicos del número áureo?

Tabla. 6

Resultados de las respuestas a la pregunta 6

\begin{tabular}{lll}
\hline Respuesta & Cantidad de estudiantes & Porcentaje (\%) \\
\hline Sí & 29 & 94 \\
No & 0 & 0 \\
Nula & 2 & 6 \\
Total & 31 & 100 \\
\hline \multicolumn{2}{c}{ Fuente: elaboración propia }
\end{tabular}

En la Tabla 6 (ver Tabla 6) se describe que un total de 29 estudiantes, representado por el $94 \%$ respondieron que los cursos de matemática en niveles superiores de la Carrera, Esta obra se comparte bajo la licencia Creative Common Atribución-No Comercial 4.0 International (CC BY-NC 4.0) 
deberían ser abordadas con la metodología usada en el curso de matemática básica fundamentada en el estudio del número áureo. Por su parte el $6 \%$ de los encuestados fue considerado con respuestas nulas ya que estas carecieron de argumentación o justificación.

\section{Conclusiones y desafíos}

El solo hecho de plantearles a los estudiantes actividades diferentes a la clase tradicional de matemáticas, conllevó a un cambio de conducta en la motivación y las actitudes favorables observadas, desde el mismo momento del planteamiento de la primera actividad. El asignarle investigar sobre la proporción aurea (tema hasta ahora desconocidos para ellos) y darle como referencia de búsqueda cápsulas audiovisuales informativas (vídeos de corta duración que llaman la atención del nativo digital) cambió el clima de aprendizaje en el aula.

Consideramos que, romper el esquema de una clase tradicional, crea una expectativa en el estudiante distinta y favorable en comparación a la que ya tiene cuando sabe que será una clase de matemáticas más, rutinaria, cuyo contenido no es significativo para él y en consecuencia no trascenderá al aula de clases. Por tanto, se logra un clima de atracción al tema tratado, por parte de los dicentes, conllevando a la generación de un aprendizaje significativo, donde además se ataca el tópico profesional de la carrera generando la fusión de la matemática con la realidad.

Analizando las seis principales preguntas realizadas en la encuesta aplicada a los 31 participantes que culminaron el curso (no todos aprobaron la asignatura pero sí asistieron hasta el último día de clases) se observa cómo la mayoría logró extraer conceptos elementales que surgieron del estudio del número áureo, entre los que destacaron: operaciones elementales, los números irracionales, proporciones, medición, sucesiones, polígonos y poliedros regulares y transformaciones geométricas (ver resultados de la pregunta 1), los cuales eran parte del programa curricular de la asignatura; sin embargo una minoría señaló que estos conceptos no eran básicos sino avanzados o difíciles de comprender y esa minoría se correspondió con los estudiantes que reprobaron la asignatura.

Es importante señalar que esta manera de impartir los contenidos básicos mediante estudio del número áureo despertó el interés en la mayoría del estudiantado quienes mostraron entusiasmo por la realización de actividades no tradicionales, en especial, por ser en la clase de matemáticas y así lo manifestaron en las justificaciones dadas en la respuesta 2 (ver resultados de la pregunta 2). Adicionalmente, en la tercera pregunta se evidencia que los estudiantes aprecian los contenidos estudiados en cuanto a la importancia que tiene la adquisición de éstos para la Carrera de Arquitectura (ver resultados de la pregunta 3).

En el mismo orden de ideas, en la pregunta 4 (ver resultados de la pregunta 4); los estudiantes, en su gran mayoría (21 estudiantes en total), manifestaron que la matemática básica abordada y apoyada en los tópicos del número áureo, permitirá valorar a la Ciencia en el ámbito futuro profesional, y un estudiante estuvo en desacuerdo con esa valoración. Entre tanto la gran mayoría de estudiantes, un $84 \%$ (ver resultados de la pregunta 5); manifestaron que los tópicos del número áureo estudiados, pueden ser de sustento en sus asignaturas prácticas de la carrera. 
Finalmente, para el ítem 6 (ver resultados de la pregunta 6); los estudiantes manifestaron en casi su totalidad (29 estudiantes), que las asignaturas de matemática superior ubicadas en los semestres subsiguientes, deberían ser abordadas considerando la metodología implementada en el curso de matemática básica, específicamente apoyada en los temas del número áureo.

De manera general estos primeros resultados dejan entrever la gran motivación que crea en el estudiantado la realización de actividades diferentes a las acostumbradas dentro del aula. Por tanto, se hace necesario incluir actividades donde el discente deje de ser considerado como un ente pasivo y mero receptor de contenidos matemáticos presentados con una tradicional formalidad y rigurosidad académica; no hay que olvidar que quienes aprenden son humanos y, por ende, se motivan, se emocionan y toman decisiones acordes con las significaciones derivadas durante la transposición didáctica; lo cual nos insta a seguir aplicando herramientas y estrategias no tradicionales y diferentes para seguir obteniendo cambios y resultados distintos a los acostumbrados (insatisfacción, cuestionamiento, bajo rendimiento, apatía, índice altos de estudiantes reprobados y deserción).

Además, debemos seguir analizando el impacto de estas actividades en el proceso educativo; como valoración general de la mejora que la herramienta proporciona en el aula; existen otras conclusiones derivada de esta misma experiencia, por ejemplo, se observó un incremento en la asistencia (sesiones de clase donde se desarrollaron las actividades del proyecto) y un mayor interés en participar en la actividad de la clase, factores que, sin duda alguna, contribuyen de manera favorable con el proceso de enseñanza y aprendizaje de las matemáticas en general.

En el futuro próximo queda mucho por investigar, aún no se conoce el impacto a mediano o largo plazo que este tipo de experiencias tuvo sobre el proceso académico seguido con este grupo particular, así como sus repercusiones en otros grupos de interés; son procesos prolongados en el tiempo, que deben seguirse aplicando, modificando y analizando de forma simultánea; cada vez que se hace algo diferente en clases de matemáticas, debemos buscar la forma de medir y valorar cómo afecta ese cambio al estudiantado, sobre todo, en su futuro desarrollo como profesional.

A manera de cierre, vale aclarar que la pretensión no es afirmar que se ha llegado a encontrar la forma de hacer que todos los estudiantes quieran aprender matemáticas; pero si consideramos que hemos dado un paso importante para liberar a las matemáticas de los cuatros paredes, llamadas aulas, en las que ha estado confinada las últimas décadas y brindarle al estudiante de hoy un camino distinto de aprendizaje. En el mismo orden de ideas, hay cuestiones que aún quedan susceptibles de ser investigadas, como por ejemplo, lo que ha fallado en esos estudiantes que no han logrado alcanzar los conocimientos necesarios pretendidos a pesar de las actividades realizadas; en ese punto, se considera que un análisis cualitativo es idóneo para abordar esas cuestiones intrínsecas al ser humano, sobre todo cuando el interés del investigador es indagar sobre las motivaciones necesarias del estudiante de hoy para aprender matemáticas. 
(s.f.).

\section{Bibliografía}

Angulo, P. J. (2006). La enseñanza de la matemática: proceso versus resultado. Educere la revista venezolana de educación, 343-345.

Banerjee, P. A. (2016). A systematic review of factors linked to poor academic performance of disadvantaged students in science and maths in schools. Cogent Education, 1-17.

Burns, D. (2011). Systemic action research: a strategy for whole system change,. Educational Action Research, 245-251.

Caballero - Jiménez, F., \& Espínola - Reyna, J. G. (2016). EL RECHAZO AL APRENDIZAJE DE LAS MATEMÁTICAS A CAUSA DE LA VIOLENCIA EN EL BACHILLERATO TECNOLÓGICO . Revista raximhai, 143-161.

Cantoral, R. (03 de Abril de 1990). Categorías relativas a la apropiación de una base de significaciones para conceptos y procesos matemáticos de la Teoría elemental de las Funciones Analíticas. Simbiosis y Predación entre las nociones de "el Prædiciere" y "lo Analítico". (Tesis Doctoral). Categorías relativas a la apropiación de una base de significaciones para conceptos y procesos matemáticos de la Teoría elemental de las Funciones Analíticas. Simbiosis y Predación entre las nociones de "el Pradiciere" y "lo Analítico”. (Tesis Doctoral). México, México D.F, México: Cinvestav.

Cantoral, R. (1995). Matemática, Matemática Escolar y Matemática Educativa. Memorias de la Novena Reunión Centroamericana y del Caribe sobre Formación de Profesores e Investigación en Matemática Educativa, (págs. 1-10).

Cantoral, R. (2002). La sensibilidad a la contradicción: Un estudio sobre la noción de logaritmo de. Acta Latinoamericana de Matemática Educativa (págs. 35-42). México: ALME.

Cantoral, R. (2011). Fundamentos y métodos de la Socioepistemología. Simposio en Matemática Educativo (págs. 22-26). México: IPN.

Cantoral, R. (2013). Teoría Socioepistemológica de la Matemática Educativa. Estudios sobre construcción social del conocimiento. Barcelona: Gedisa.

Cantoral, R., \& Farfán, R. M. (2003). Matemática Educativa: Una visión de su evolución. Revista Latinoamericana de Investigación en, 27-40.

Cantoral, R., Reyes-Gasperini, D., \& Montiel, G. (2014). Socioepistemología, Matemáticas y Realidad. Revista Latinoamericana de Etnomatemática, 91-116.

Cantoral, R., Reyes-Gasperini, D., \& Montiel, G. (2014). Socioepistemología, Matemáticas y Realidad. Revista Latinoamericana de Etnomatemática, 91-116.

Cantoral, R., Reyes-Gasperini, D., \& Montiel, G. (2014). Socioepistemología, Matemáticas y Realidad. Revista Latinoamericana de Etnomatemática, 91-116. 
Carraher, T. N. (1982). Na vida, dez, na escola, zero: os contextosculturais da aprendizagem da matemática. ("A"in life, "F"in the school: the cultural contexts of learningmathematics.). . Cuaderno de pesquisa, 79-86.

Cerda, G. \&. (2018). Algunos factores asociados al desempeño académico en matemáticas y sus proyecciones en la formación docente. . Educação e Pesquisa, 1-19.

Corbalán, F. (2012). La proporción áurea: el lenguaje matemático de la belleza. Barcelona: RBA Coleccionables .

Cordero Osorio, F., \& Silva-Crocci, H. (2012). MATEMÁTICA EDUCATIVA, IDENTIDAD Y LATINOAMÉRICA: EL QUEHACER Y LA USANZA DEL CONOCIMIENTO DISCIPLINAR. Revista Latinoamericana de Investigación en Matemática Educativa, 295318.

Delgado, I. E. (2017). Ansiedad matemática en estudiantes universitarios de Costa Rica y su relación con el rendimiento académico y variables sociodemográficas. Propósitos y Representaciones, 275-324.

Doczi, G. (1994). The Power of Limits: Proportional Harmonies in Nature, Art, and Architecture. Boston : ShambhalaPublications.

Fajardo Bullón, F., Maestre Campos, M., Felipe Castaño, E., León del Barco, B., \& Polo del Río, M. I. (2017). ANÁLISIS DEL RENDIMIENTO ACADÉMICO DE LOS ALUMNOS DE EDUCACIÓN SECUNDARIA OBLIGATORIA SEGÚN LAS VARIABLES FAMILIARES . Educación XX1, 209-232.

Ghyka, M. (1977). The Geometry of Art and Life. Dover Publications.

Hemenway, P. (2008). El código secreto. La misteriosa fórmula que rige el arte, la naturaleza y la ciencia. Barcelona: Evergreen.

Livio, M. (2002). The golden ratio : the story of phi, the world's most astonishing number. New York: Broadway Books.

Mora, D. (2009). Didáctica de las Matemáticas. Desde una persectiva crítica, investigativa, colaborativa y transformadora. La Paz: Fondo Editorial Ipasme.

Nuria Gil, L. J. (2005). El dominio afectivo en el aprendizaje de las matemáticas. Revista Iberoamericana de Educación Matemática, 15-32.

Padrón, O. J. (2016). ¿Qué dicen los docentes paraguayos en cuanto al afecto en el aprendizaje de la Matemática?: Una mirada desde el Curso Nanduti . Revista Iberoamericana de Educación Matemática, 24-43.

Perez, A. G. (2009). Guía metodológica para anteproyectos de investigación. Caracas: Fedupel.

PISA. (2016). Estudiantes de bajo rendimiento. Paris: OCDE.

Prensky, M. (2011). Enseñar a Nativos Digitales. Bogotá: EDICIONES SM. 
Ramohapi, S. M. (2017). Investigating Factors Contributing To Learner Performance in Mathematics: A Case Study of Some Selected Schools in Motheo District. International Journal of Educational Sciences, 445-451.

Salinas, P. \&. (2009). Hacia un nuevo paradigma en la enseñanza del cálculo dentro de una institución educativa. Revista Latinoamericana de Investigación en Matemática Educativa, 355-382.

Ventura, C. (30 de Agosto de 2016). Red de Cerebros. Obtenido de Fibonacci y el Número de Oro: https://www.youtube.com/watch?v=8bCYiUIlF2k

Zemelman, S. H. (1998). Best Practice: New Standards for Teaching and Learning in America's Schools. Illinois: Editorial Hinemann. 